\title{
Bilateral Simultaneous T3/T4 Endoscopic Thoracic Sympathectomy for Treatment of Primary Palmar and Axillary Hyperhidrosis
}

\author{
Mohamed Lasheen, MD. \\ Department of General Surgery, Ain Shams University, Cairo, Egypt.
}

Objective: This study was conducted to evaluate the safety, and complications of bilateral simultaneous endoscopic thoracic sympathectomy by resection of T3/T4 sympathetic ganglia in treatment of primary palmar and axillary hyperhidrosis.

Patients and methods: This prospective study was conducted in General Surgery Unit at Ain Shams University Hospitals in the period from May 2012 to June 2014 on twenty patients. All cases underwent thoracoscopic sympathectomy of T 3, 4 ganglia on the right \&left sides at the same setting. In this prospective interventional study, we had 20 patients with severe primary hyperhidrosis with failed medical treatment who underwent bilateral simultaneous Endoscopic Thoracic Sympathectomy (ETS).

Results: Of 20 patients there were 13 males (65\%) and 7 females (35\%). The mean age was $23.9 \pm 7.8$. The mean operating time was $76.2 \mathrm{~min} . \pm 21.4 \mathrm{~min}$. all cases were completed laparoscopically and there were no serious intraoperative complications.

The average length of hospital stay was 2.8 days (range: 2-5 days). The average postoperative follow-up period was 14.6 months (range: 6-20 months).

Residual pneumothorax occurred in 3 patients (15\%), Surgical emphysema on either side was seen in the early postoperative period in 3 patients (15\%).

The postoperative intercostal pain was observed in all cases (100\%). most patients (19/20: 95\% of patients) experienced compensatory sweating of varying degrees in other parts of the body, which was bilateral and symmetrical, most commonly at the lower back.

Excessive hand dryness was reported in 8 patients (40\%), one of them had to use moisturizing hand cream daily.Patients' Satisfaction with the overall results of the operation were assessed .Only one patient (5\%) stated that they were 'dissatisfied' and regretted having undergone the operation

Conclusion: ETS with T3/T4 ganglia resection is a safe method for patients complaining of severe palmar or combined palmar and axillary hyperhidrosis . From the results of this study, we recommend using smaller ports and performing a more limited approach (T3 or T4) to patients with moderate primary hyperhidrosis symptoms to avoid dissatisfaction due to severe compensatory sweating and palmar overdryness.

Key words: Palmar hydrosis, sympathectomy, surgery, thoracoscopy.

\section{Introduction:}

Hyperhidrosis is a condition of sweating greater than necessary for the maintenance of normal body thermoregulation. It may develop secondary to a variety of medical disorders or it may be primary. ${ }^{1}$

For severe primary hyperhidrosis, medical management is often frustrating leading many patients to try multiple unsuccessful treatment modalities, ${ }^{2}$ Over the past couple of decades endoscopic transthoracic sympathectomy (ETS) has gained popularity as a minimally invasive endoscopic approach in performing thoracic sympathectomy. ${ }^{3}$ Compensatory sweating is the most common side effect, and was thought to be due to a thermoregulatory 
mechanism but it is believed now to be a reflex response. ${ }^{1}$

The aim of this study is to evaluate the safety and complications of bilateral simultaneous endoscopic thoracic sympathectomy by resection at levels T3/T4 sympathetic ganglia in treatment of primary palmar and axillary hyperhidrosis.

\section{Patients and methods:}

This prospective study was conducted in General Surgery Unit at Ain Shams University Hospitals between May 2012 to June 2014. It was carried out on twenty patients, the characters of the patients are listed in Table (1).

Patients with secondary hyperhidrosis, recurrent hyperhidrosis after surgery, previous major thoracic surgery and those who were unfit for general anaesthesia were excluded.

All patients were subjected to clinical assessment by history taking and examination to avoid patients with secondary hyperhidrosis. Preoperative investigations were done to all patients which include $\mathrm{CBC}$, bleeding profile, liver enzymes, renal function tests and ECG. Chest X-ray and pulmonary function test were also done to exclude pulmonary pathology.

All cases underwent endoscopic thoracoscopic sympathectomy of $\mathrm{T} 3,4$ ganglia on the right \&left sides at the same setting. A postoperative chest $\mathrm{x}$-ray was done before discharge. Patients were followed-up for one year.

\section{Surgical technique:}

The procedure was done under general anaesthesia with double lumen endotracheal tube to allow single lung ventilation. All patients were positioned in thoracotomy position Figure (1).

Pneumothorax was achieved with Verres needle using $\mathrm{CO} 2$ insufflations under continuous pressure of $14 \mathrm{mmHg}$.three ports are introduced; the first $10 \mathrm{~mm}$ (for 30 degree Camera) in the fifth intercostal space midaxillary line the second one $5 \mathrm{~mm}$ in $3 \mathrm{rd}$ or 4th spaces anterior axillary line and the third10 $\mathrm{mm}$ in 6 th space anterior axillary line.
Using monopolar diathermy coagulation on a hook, excision of third \& forth ganglia are done Figure (2). The rami communicans at the curves of ribs 3, 4 were also severed.

Patient was then repositioned and the procedure is repeated on the contralateral side.

Patients received oral analgesic agents for adequate pain control. All patients were discharged after exclusion of pneumothorax or any other serious complication \& removal of the intercostal tube. Patients came for follow-up in outpatient clinic after 10 days for removal of stitches. All patients were advised preoperatively that follow-up was necessary. after 10 days, 1 month, 6 months and 1 year.

All the patients were assessed for: Operative time ,hospital stay , intraoperative complications postoperative complications and degree of satisfaction which were assessed by simplified questionnaires at 1 month, 6 months and 1 year follow-up OPC visits. Degree of satisfaction was divided into four grades: very satisfied, satisfied, partially (not very) satisfied and dissatisfied. This classification regarding the satisfaction with the procedure was obtained spontaneously during a clinical interview with the patients.

\section{Results:}

In this prospective interventional study, we had 20 patients with severe primary hyperhidrosis with failed medical treatment who underwent bilateral simultaneous Endoscopic Thoracic Sympathectomy (ETS).

Operative results

Bilateral thoracic sympathectomy operations (T3-T4) were successfully completed in all patients under video-assisted thoracoscopic guidance. The mean operating time was $76.2 \mathrm{~min} . \pm 21.4 \mathrm{~min}$.

There was no conversion to open thoracotomy, no vascular injury and no severe pleural adhesions. There were no serious intraoperative complications, such as massive hemorrhage, arrhythmia or sudden cardiac arrest.

The average length of hospital stay was 2.8 days (range: 2-5 days). The intercostal tubes on both sides were removed on the 


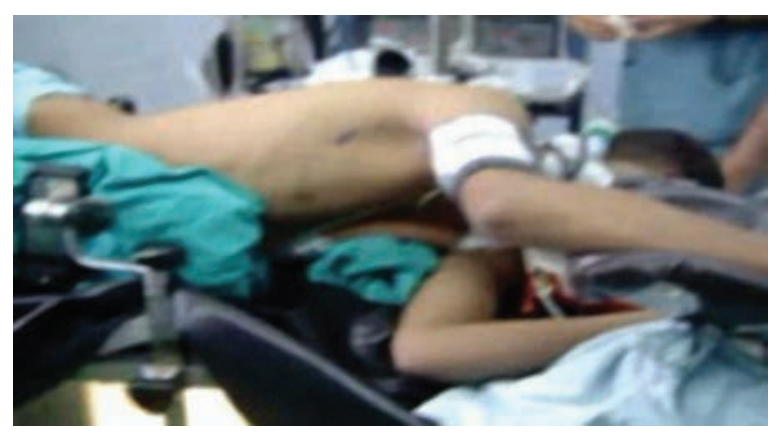

Figure (1): Patient position.

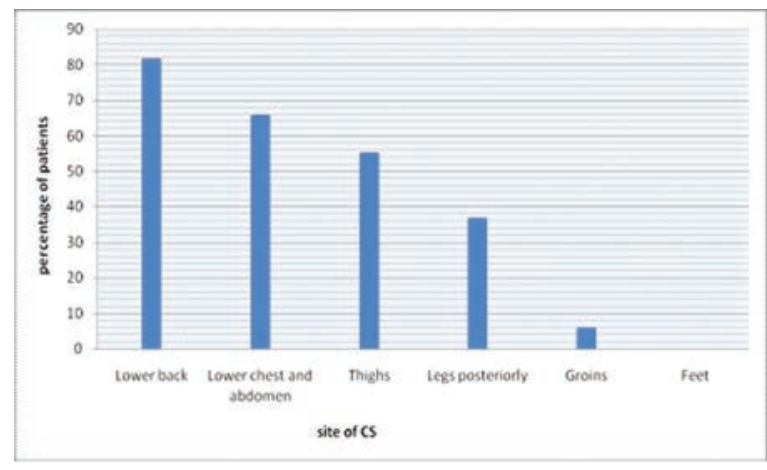

Figure (3): Locations of compensatory sweating

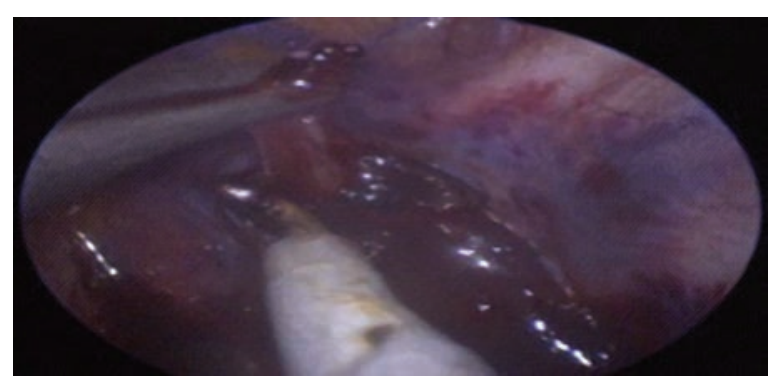

Figure (2): Excision of third ganglion.

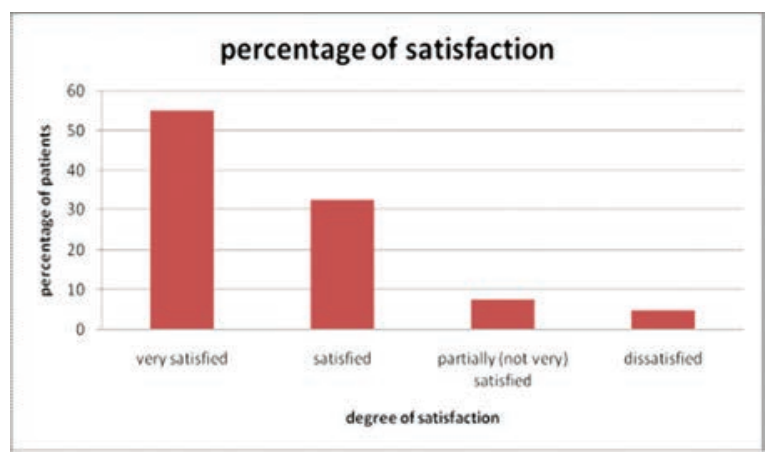

Figure (4): Degree of satisfaction.

Table (1): Characters of the patients.

\begin{tabular}{|l|l|}
\hline age & Mean age of $23.9 \pm 7.8$ \\
\hline sex & 13 males $(65 \%)$ and 7 females $(35 \%)$ \\
\hline Palmar hydrosis & 20 patients $(100 \%)$ \\
\hline Palmar + axillary hydrosis & 8 patients $(40 \%)$ \\
\hline +ve family history & 3 patients $(15 \%)$ \\
\hline
\end{tabular}

Table (2): Efficacy rate at different sites in the study.

\begin{tabular}{|l|l|}
\hline \multicolumn{1}{|c|}{ Site of sweating } & \multicolumn{1}{c|}{ Efficacy rate } \\
\hline Palmar & $100 \%$ \\
\hline Axillary & $100 \%$ \\
\hline Plantar & $78 \%$ \\
\hline
\end{tabular}

second post operative day after chest X-ray confirming the absence of pneumothorax.

The average postoperative follow-up period was 14.6 months (range: 6-20 months). There were two cases of dropouts during the study.

There was no mortality in this study, and no Horner syndrome was observed.

Residual pneumothorax occurred in 3 patients $(15 \%)$ as seen in the postoperative chest radiography, complete resolution occurred in all of them without intervention.

Surgical emphysema on either side was seen in the early postoperative period in 3 patients (15\%).

The postoperative intercostal pain was experienced by all cases (100\%) which was treated by opioids (Pethidine) for only 24 hours after surgery. Later on it was successfully managed with NSAIDs. 
Mean basal heart rate before the procedure was $78.7 \mathrm{bpm} \pm 8.5$ while mean basal heart rate 10 days after was $74.5 \mathrm{bpm} \pm 10.3$. There was about $5.3 \%$ decrease in the postoperative mean

Efficacy rate at different sites are shown in Table (2).

Most of the patients (19/20; 95\%) experienced compensatory sweating of varying degrees in other parts of the body, which was bilateral and symmetrical, most commonly at the lower back. The locations of compensatory sweating are summarized in Figure (3).

Compensatory sweating was graded as: Grade 1: No CS, Grade 2: mild (minor), Grade 3: moderate (embarrassing) and Grade 4: severe (disabling or incapacitating). The percentage of compensatory hyperhidrosis in the patients according to these grades was: Grade 1 in 1 patients (5\%), Grade 2 in 12 patients $(60 \%)$, Grade 3 in 6 patients $(30 \%)$, and Grade 4 in only one patient (5\%).

Excessive hand dryness was reported in 8 patients $(40 \%)$, especially during winter. Only one patient $(5 \%)$ stated that her hands became too dry to be accepted and had to use moisturizing hand cream daily.

Patients' Satisfaction with the overall results of the operation was assessed by simplified questionnaires at 1 month, 6 months and 1 year follow-up OPC visits. Regarding the degree of satisfaction indicated by the patients at the end of the study, Only one patient $(5 \%)$ stated that she were 'dissatisfied' and regretted having undergone the operation Figure (4).

\section{Discussion:}

This study involved a series of 20 patients of palmar with or without associated axillary hyperhidrosis who underwent video-assisted bilateral endoscopic thoracic sympathectomy (ETS). There is noticeable paucity of the series that investigate the resection of T3/T4 ganglia in literature. So, in this study we have chosen to study sympathectomy at level T3/ T4 and to evaluate the safety or (outcome) and the incidence of complications.

In this study the mean operating time was relatively longer (76.2 $\pm 21.4 \mathrm{~min}$.) compared to other studies as that done by Katara $^{4}$ (35 min.) and Stefaniak ${ }^{5}$ that studied T3/T4 clipping (57 min.). This is mostly because we used the lateral position that requires repositioning of the patient during the procedure in addition to the 2 ganglia resection technique that consumes more time .

In this study the average length of hospital stay was 2.8 days (range: $2-5$ days) which was slightly higher than that done by Stefaniak where it was 1.3 days. Whereas it was similar to the study done by Çubuk ${ }^{6}$ in which was 2.7 days. ${ }^{2-8}$

we had no cases of hemothorax in the 20 patients $(0 \%)$. This was similar to studies by Kim, ${ }^{7}$ and Mahdy. ${ }^{8}$

Very few recent series encountered Horner's syndrome as in study done by Yazbek $^{9}$ where only 1 patient (out of 60) had transient Horner's syndrome mostly due to heat transmission with adhesiolysis. In this study, the incidence of Horner's syndrome was $0 \%$ which was similar to most of the studies in recent literature.

The most common complication is pneumothorax as mentioned by $\mathrm{Liu}^{10}$ who reported 3 patients $(2.1 \%)$ with unilateral pneumothorax not requiring drainage. While Chen, ${ }^{11}$ reported 5 patients $(11 \%)$ with unilateral pneumothorax and none of them required chest drainage,we encountered 3 cases $(15 \%)$ with pneumothorax in the early postoperative period,3 out of them were minimal and insignificant and only one case (5\%) required chest drainage for 4 days.

Surgical emphysema is infrequently reported in literature although it is not uncommon. Chang 12 reported that $32.5 \%$ of their patients developed subcutaneous emphysema, while in a study done by Moya ${ }^{13}$ surgical emphysema was found in about $1 \%$ of patients.

The rate of surgical emphysema in this study was 3 patients (15\%). They were detected both clinically and radiologically .All cases were managed conservatively and it resolved completely within 2 weeks.

Compensatory sweating (CS) of the trunk and lower limbs In a study by Mahdy 
et al ${ }^{14}$ was $60 \%$ with $\mathrm{T} 2,45 \%$ with $\mathrm{T} 3$ and $10 \%$ with T4. While Yang 15 showed that the overall incidence of CS was $44.7 \%$ after T4 sympathectomy and $70 \%$ with T3 sympathectomy.

In the present study where T3/T4 sympathectomy was performed, the total incidence of compensatory sweating of any degree was $95 \%$. The mild form was detected in $60 \%$; the moderate form was detected in $30 \%$ and the severe disabling form was detected in only one case .

Excessive palmar dryness was reported by Chang 12 in $8.6 \%$ for T4 sympathectomy, $39.7 \%$ for $\mathrm{T} 3$, and $36 \%$ for $\mathrm{T} 2$. While $\mathrm{Liu}^{9}$ noticed dry hand in group T4 (1.4\%) and, in group T3 (12.9\%). In this study where T3/ T4 sympathectomy was performed, $40 \%$ of patients (8 cases) complained of excessive hand dryness that was tolerable to most except for one of them $(5 \%)$. These results are not far from T3 sympathectomy results mentioned in other series and this slightly higher incidence may be due to the combined T3/T4 approach.

According to Chwajol et al., 200916 satisfaction rates after endoscopic thoracoscopic sympathectomy reported was $90 \%$.while Loscertales ${ }^{17}$ in 2004 in a study of 226 patients, found that $97 \%$ of their patients said they would undergo the operation again. in this study, Only one patient (5\%) stated that she were 'dissatisfied' and regretted having undergone the operation.

\section{Conclusion:}

From this study we have concluded that endoscopic thoracoscopic sympathectomy with T3/T4 ganglia resection is effective and safe method for patients complaining of severe palmar or combined palmar and axillary hyperhidrosis with acceptable side effects leading to high degrees of patient satisfaction and improvement in the quality of life. From the results of this study, we recommend using ports $5 \mathrm{~mm}$. to reduce postoperative chronic pain and incidence of surgical emphysema. owing to the high incidence of compensatory sweating and hand over dryness with T3/T4 resection, we recommend performing a more limited approach (T3 or T4) to patients with moderate primary hyperhidrosis symptoms to avoid dissatisfaction due to severe compensatory sweating and palmar over dryness.

\section{Reference:}

1- Cerfolio RJ, De Campos JRM, Bryant AS, Connery CP, Daniel L. Miller DL, Malcolm M. DeCamp MM, McKenna RJ, Krasna MJ: The Society of Thoracic Surgeons Expert Consensus for the Surgical Treatment of Hyperhidrosis. Ann Thorac Surg 2011; 91: 1642-1648.

2- Jeganathan R, Jordan S, Jones M, Grant S, Diamond O, McManus K, Graham A, McGuigan J: Bilateral thoracoscopic sympathectomy: Results and long-term follow-up. Interactive Cardio Vascular and Thoracic Surgery 2008; 7: 67-70.

3- El-Anwar AM, Nasser A, Adel H: Minimally invasive surgery for primary hyperhidrosis. Kasr El Aini Journal of Surgery 2009; 10(3): 45-52.

4- Katara AN, Domino JP, Cheah WK, So JB, Ning C, Lomanto D: Comparing T2 and T2T3 ablation in thoracoscopic sympathectomy for palmar hyperhidrosis: A randomized control trial. Surg Endosc 2007; 21: 1768-1771.

5- Stefaniak TJ, Ćwigoń M: Long-term results of thoracic sympathectomy for primary hyperhidrosis. Polski Przeglad Chirurgiczny 2013; 85(5): 247-252.

6- Çubuk S, Yücel O, Karataş O, Kavaklı K, Çaylak H, Gürkök S, Sapmaz E, Iş̧k H, Gözübüyük A, Genç O: Analysis of 54 patients who underwent thoracoscopic sympathectomy. J Ann Eu Med 2013; 1(1): $1-3$

7- Kim WO, Kil HK, Yoon KB, Yoon DM, Lee JS: Influence of T3 or T4 sympathectomy for palmar hyperhidrosis. The American Journal of Surgery 2010; 199: 166-169.

8- Mahdy T, Youssef T, Abd Elmonem H, Omar W, Abd Elateef A: T4 sympathectomy for palmar hyperhidrosis: Looking for the right operation. Surgery 2008; 143: 784-789.

9- Yazbek G, Wolosker N, Kauffman P, De Campos JRM, Leão PP, Jatene FB: Twenty months of evolution following sympathectomy on patients with palmar hyperhidrosis: Sympathectomy at the T3 level is better than at the T2 level. Clinics 2009; 64(8): 743-749.

10- Liu Y, Yang J, Liu J, Yang F, Jiang G, Li 
J, Huang Y, Wang J: Surgical treatment of primary palmar hyperhidrosis: A prospective randomized study comparing T3 and T4 sympathectomy. European Journal of Cardio-thoracic Surgery 2009; 35: 398-402.

11- Chen YB, Wu YE, Yang WT, Shi L, Guo XF, Xu ZH, Qian YY: Uniportal versus biportal video-assisted thoracoscopic sympathectomy for palmar hyperhidrosis. Chin Med J 2009; 122(13): 1525-1528.

12- Chang YT, Li HP, Lee JY, Lin PJ, Lin CC, Kao EL, Chou SH, Huang MF: Treatment of palmar hyperhidrosis T4 level compared with T3 and T2. Ann Surg 2007; 246: 330-336.

13- Moya J, Ramos R, Morera R, Villalonga R, Perna V, Macia I, Ferrer G: Thoracic sympathicolysis for primary hyperhidrosis, A review of 918 procedures. Surg Endosc 2006; 20: 598-602

14- Mahdy T, Youssef T, Abd Elmonem H, Omar
W, Abd Elateef A: T4 sympathectomy for palmar hyperhidrosis: Looking for the right operation. Surgery 2008; 143: 784-789.

15- Yang J, Tan JJ, Ye GL, Gu WQ, Wang J, Liu YG: T3/T4 thoracic sympathectomy and compensatory sweating in treatment of palmar hyperhidrosis. Chin Med J (Engl) 2007; 120: 1574-1577.

16- Chwajol M, Barrenechea IJ, Chakraborty S, Lesser JB, Connery CD, Perin NI: Impact of compensatory hyperhidrosis on patient satisfaction after endoscopic thoracoscopic sympathectomy. Neurosurgery 2009; 64: 511-518.

17- Loscertales J, Arroyo TA, Congregado LM et al: Thoracoscopic sympathectomy for palmar hyperhidrosis. Immediate results and postoperative quality of life [in Spanish]. Arch Bronconeumol 2004; 40: 67-71. 